thelzellen, die Muskelfasern und Parenchymzellen, ferner die Elemente der Endothelien, die Blut- und Lymphgefässe. Die Resorption des Fettes geschieht von diesem Reticulum aus und durch seine Vermittelung gelangt es in die Lymph- und wahrscheinlich auch in die Blutgefässe. - Alles in allem genommen scheint das Reticulum des Verfassers mit dem, was man sonst allgemein Intercellularsubstanz nennt, identisch zu sein." (Ref.)

(Aus dem physiologischen Institut der Universität Jena.)

\title{
Die Wirkung der Kalium- und Natrium-Salze auf die glatte Muskulatur verschiedener Thiere.
}

\author{
Von \\ Dr. med. Otto Flöel
}

in Jena.

Hierzu 10 Holzschnitte.

Als die Untersuchungen Nothnagel's iber chemische Reizung glatter Muskeln veröffentlicht waren, stellte ich auf Veranlassung des Herrn Hofrath Preyer und unterstiitzt von demselben im hiesigen physiologischen Institut ähnliche Versuche an.

Die älteste Angabe über chemische Reizung glatter Muskeln findet sich in dem Handbuche der Physiologie von Johannes M ülle ${ }^{1}$ ). Es heisst da, dass bei chemischer, mechanischer, electrischer Reizung der Darm sich contrahirt. Spina beobachtete am Fliegendarm nach Application von 0,3-procentiger Kochsalzlösung Contraction des Darmes verbunden mit einer Anschwellung. des Epithels, nach Anwendung von 5-procentiger Atropinlösung Dilatation des contrahirten Darmes mit Absehwellung der Epithe-

1) J. Müller, Handbuch der Physiologie des Menschen. Bd. I, p. 506, 3. Auflage. 
lien und wahrnehmbarer Contraction der Längsmuskulatur ${ }^{1}$ ). Neue Thatsachen insbesondere über die Wirkung der Kalium- und Natriumverbindungen als chemischer Reize für die glatten Muskeln bringen die Untersuchungen von Nothnagel ${ }^{2}$ ) und die Mittheilungen von Bardeleben ${ }^{3}$ ), nachdem schon früher im hiesigen physiologischen Institut von R. Wernicke ${ }^{4}$ ) die Verschiedenbeit der Wirkung jener Verbindungen anf contractiles, embryonales Gewebe, nämlich auf das embryonale Herz, ehe die Querstreifung seiner Muskelfasern deutlich wurde, dargethan war.

An die Untersuchungen von Nothnagel und Bardeleben schliessen sich meine Versuche direct an. Sie sollten in erster Linie feststellen, wie die beim Menschen, bei der Katze und hauptsächlich beim Kaninchen beobachtete Reaction auf Application von Kalium- und Natriumsalzen bei anderen Thieren sich gestaltet. Das Rubidiumchlorid, Lithiumchlorid und Ammoniumchlorid, die ich anfangs neben dem Nitrat, Sulfat, Chlorid des Kalium und Natrium anwandte, liess ich später beiseite. Die Wirkung des Rubidium- und Lithium-Chlorid gleicht der Wirkung der Kalisalze, die des Salmiaks nähert sich der der Natriumsalze. Die Versuchsanordnung war in der Regel die von Sanders und van Braam Houckgeest eingefúhrte. Das Abdomen des lebenden Thieres wurde in einem geräumigen 0,6-procentigen Kochsalzbade geöffnet und bei einer der Blatwärme des Thieres entsprechenden Temperatur beobachtet. Der Kopf wurde durch eine einfache Vorrichtung iiber Wasser gehalten oder nach der Tracheotomie

1) A. Spina, Unters. über die Mechanik der Darm* und Hautresorption LXXXIV. Band der Sitzb. d. k. Akad, der Wissensch. III. Abth. Juli-Heft. Jahrg. 1881.

2) H. Notbnagel, Zur chem. Reizung der glatten Muskeln. Virchow's Archiv f. pathol. Anatomie und Physiologie und für klin. Medicin. Bd. 88, 1882. - Ueber Einwirkung des Morphin auf den Darm Bd. 89, 1882.

3) K. Bardeleben, Die Einwirkung von Kali- und Natronsalzen auf die Muskeln des menschlichen Darmes. Sitzungsbericht der Jenaischen Gesellschaft für Medicin und Naturwissenschaft. Jahrgang 1882. - Tageblatt der 55. Versammlung deutscher Naturforscher und Aerzte in Eisenach. 1882. pag. 244.

4) Rob. Wernicke, Zur Physiologie des embryonalen Herzens. Jena, Fischer, 1876 S. 21-24. 33. Auch in Preyer's Sammlung physiologischer Abhandlungen I. Bd., S. 260. Vgl. Preyer, Spec. Physiol. des Embryo, S. 33. 
das ganze Thier untergetaucht. Die Thiere wurden bisweilen ätherisirt, ohne dass ich dann eine Aenderung der Versuchsresultate bemerkt hätte. Die Reizung geschah dureh Salze in Substanz, oder es wurde eine Lösung so von aussen auf die Darmwand gebracht, dass die betreffende Darmschlinge während der Reizung ausser Wasser gehalten wurde. Letztere Reizmethode sollte die Fehlerquelle vermeiden, die durch die verschiedene Löslichkeit der Salze bedingt wird. Die Ergebnisse iiber Energie und Dauer der Contraction mögen deshalb werthvoller sein als bei der Reizung mit ungelösten Substanzen. Die Contraction zu localisiren ist natirlich die letztere Methode die geeignetere. Ich benutzte 54 Versuchsthiere. Es waren: Kaninchen, Katze, Meerschweinchen, Ratte, Maus, Hund, Huhn, Frosch.

Die an diesen Thierarten gemachten Beobachtungen ergaben folgendes.

Am Kaninchen stellte ich nur wenige Versuche an. Es handelte sich nicht darum, Neues zu finden, sondern bekannte und wohlbeschriebene Experimente zu wiederholen. „Die Wirkung der Kalisalze", so heisst es in der Mittheilung von Nothnagel, ,unterscheidet sich wesentlich von derjenigen der Natronsalze, wenn man die Aussenfläche des lebenden Darmes mit denselben berührt. Wenn man mit einem Kalisalze irgend eine Stelle des Darmes, sei es des Dünn- oder des Dickdarmes, berührt, so erfolgt eine starke Contraction der Musculatur, welche auf die Stelle der Berührung beschränkt bleibt oder auch den Darm an der betreffenden Stelle ringförmig umgebend einschnürt. Die Berührung mit einem Natronsalz erzeugt eine Contraction, welche nicht auf die Berührungsstelle bescbränkt bleibt, sondern über mehrere Centimeter weit sich erstreckt, und zwar ausnahmslos immer und nur in der Richtung nach aufwärts, nach dem Pylorus zu." Am Magen und an der Blase erzeugen Natrium- wie Kaliumsalze immer nur eine locale Contraction, die bei den ersteren schwächer ist. Nachdem ich die von Nothnagel beschriebenen Erscheinungen im Experiment gesehen hatte und zwar in einer Weise, dass ich jede Einzelheit bestätigen kann, stellte ich einige Versuche mit gelösten Salzen (25-procentige Lösung 1 Tropfen $1 / 4$ bis $1 / 8$ Minute) an. Es zeigte sich, dass das Kalium-Chlorid und -Sulfat kräftiger wirkte als die entsprechenden Natriumsalze, ohne dass deshalb die Contraction länger angedauert hätte. Sie versehwand am Magen und 
Dünndarm innerhalb 2 bis 7 Minuten, am Coecum und Dickdarm innerhalb 3 bis 15 Minuten. Die Kaliumcontraction erstreckte sich gewöhnlich gleichmässig über den Umfang des Darmes; bisweilen war sie an der Reizstelle stärker. Die Breite des Contractionsringes betrug immer unter $1 \mathrm{~cm}$ und entsprach genau der Reizstelle. Die Natriumeontraction war stets gleichmässig circulär; die Breite des Contractionsringes war gewöhnlich grösser als bei den Kalisalzen und betrug am Dündarm stets uber $1 \mathrm{~cm}$, in der Regel 2 bis $5 \mathrm{~cm}$. Die Contraction erstreckte sich bisweilen mehr nach aufwärts, öfter gleichmässig nach oben und unten von der Reizstelle aus. Der der Reizstelle entsprechende Theil des Contractionsringes entstand bei den Natriumsalzen bisweilen zuletzt und persistirte länger als der übrige Theil, cf. Fig. 1.

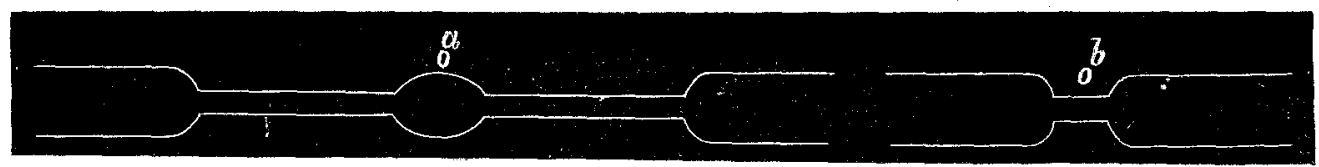

Fig. I.

a soll ein Stück Dünndarm zu Anfang, b zu Ende der Contraction darstellen nach Reizung mit Natriumchlorid.

Versuche an der Katz e ergeben nach Noth nagel qualitativ im Wesentlichen genau dieselben Erscheinungen wie beim Kaninchen, und die Uebereinstimmung tritt am deutlichsten am Dickdarm hervor. Am Dünndarm wirken die Natriumsalze bisweilen nicht, wenn sie aber wirken, dann erfolgt auch wie beim Kaninchen eine aufsteigende Zusammenziehung, nur schwächer als bei diesem. Bêi den Versuchen, die ich mit den Salzen in Substanz anstellte, bemerkte ich am Duodenum ebenfalls die aufsteigende Natriumcontraction oder wenigstens eine wellige Bewegung oberhalb der localen Contraction. Am übrigen Dünndarm und am Dickdarm gelang es mir nicht, die qualitativ verschiedene Wirkung der Kalium- und Natriumsalze zu sehen. Es zeigte sich eine schmale Einschnürung, die sich mehr oder weniger über die Peripherie des Darmes verbreitete. Die Kaliumsalze wirkten viel kräftiger als die Natriumsalze. Bei erstern genïgte eine momentane Berïhrung, bei letzteren war eine Reizung von 10 bis 15 Secunden nothwendig, um eine Wirkung hervorzurufen. Am Coecum kam die Natriumcontraction stets einige Millimeter oberhalb der Reizstelle zu Stande. 
Eindeutige Resultate erhielt ich an einer ausgewachsenen Katze, deren Duodenum und übrigen Dünndarm ich mit Lösung reizte.

Der Darm wurde genan horizontal ausser Wasser genommen, ein Tropfen einer 20-procentigen Lösung mit der Pravaz'sehen Spritze auf eine genau angemerkte Stelle gebracht und nach $1 / 4$ Minute wieder untergetaucht. Die Contractionsdaner betrug bei Kalium- wie Natriumsalzen 2 bis 7 Minuten, die Breite der Contraction im Mittel $1 \mathrm{~cm}$. Die Contraction erstreckte sich bei den Natriumsalzen über einen grösseren Theil der Peripherie als bei den Kaliumsalzen; sie entsprach bei letzteren genau der Reizstelle, bei den ersteren lag die Mitte der contrahirten Stelle $1 / 2$ bis $1 \frac{1}{2}$ Centimeter höher als die Reizstelle (Fig. 2).

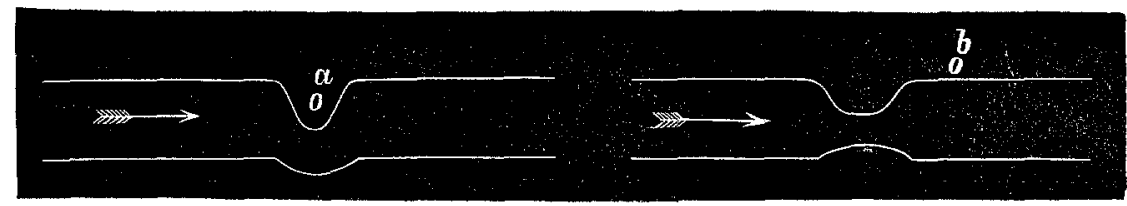

Fig. 2.

Die Figur 2 bezeichnet ein Stiick Duodenum, in a nach Reizung mit Kaliumchlorid, in b nach Reizung mit Natriumchlorid. Die Pfeile bezeichnen hier wie in den folgenden Figuren stets die Richtung, in welcher der Darminhalt peristaltisch fortbewegt wird (vom Pylorus zum Anus).

Bei den Reizversuchen am Meerschweinchen mit Lösungen der Kalium- und Natriumsalze (20 procent. 1 Tropfen $1 / 4$ Minute) trat sofort starke Contraction ein. Die Dauer derselben betrug am Magen im Durehschnitt 3 Minuten, am Duodenum 1/2, am übrigen Dünndarm $2 \frac{1}{2}$, am Coecum 7-15, am Dickdarm 3-14, an der Harnblase 7, an der Samenblase 2 Minuten. Die Contraction auf Natriumsalze war stets weniger scharf begrenzt und weniger energisch, als die der Kaliumsalze. Auffällig war am Dünndarm, besonders am Duodenum, eine grössere Längsausdehnung der Contraction nach Reizung mit Natriumsalzen. Die Länge der Zusammenziehung betrug hier $1 \frac{1}{2}$ bis 8 , in der Regel utber $2 \mathrm{~cm}$, von der Reizstelle ungefähr gleichweit nach aufwärts und abwärts; bei den Kaliumsalzen betrug sie $1 / 2$ bis $2 \mathrm{~cm}$. Die Natriumeontraction erstreckte sich gleichmässig iiber die ganze Peripherie des Darmes, die Kaliumcontraction war in der Regel an der Reizseite stärker. 
In Bezug auf die Localisation der Contraction waren die Resultate der Reizung mit Lösung gerade beim Meerschweinchen sehr wenig ausreichend, die Anwendung der Salze in Substanz brachte hingegen übereinstimmende Ergebnisse. Die Berührung dauerte $1 / 4$ bis $1 / 8$ Minute, am Coecnm war sie bisweilen nur momentan. Bei längerer Berührung fiel die Contraction wenig energischer aus als bei kuirzerer. Am Magen erfolgte eine mehr oder minder starke, bis $2 \mathrm{~cm}$ breite, mehr oder weniger peripher ausgedehnte, dem Reizpunkte entsprechende Contraction. Die Contraction danerte ungefähr 3 Minuten.

Am stärksten wirkte $\mathrm{Na}^{2} \mathrm{CO}^{3}$, es folgen absteigend $\mathrm{KNO}^{3}$, $\mathrm{KCl}, \mathrm{K}^{2} \mathrm{CO}^{3}, \mathrm{NaCl}, \mathrm{NaNO}^{3}$.

Am Duodenum dauert die Contraction 1-2 Minuten an. Bei den Natriumsalzen erstreckt sie sich oft von der Reizstelle $1 \frac{1}{2}$ bis $31 / 2 \mathrm{~cm}$ aufwärts, häufiger ist sie local, oberhalb entsteht eine Wellenbewegung, selten bleibt es allein bei der localen Contraction. Die Einschnürung erstreckt sich stets gleichmässig über den ganzen Umfang des Darmes. Die Ka-Contraction bleibt in Bezug auf die Darmaxe stets local, ist intensiver als die Natriumcontraction, und verbreitet sich gar nicht über den Umfang oder doch nicht so gleichmässig, wie dies bei den Na-Salzen der Fall ist.

Die Contractionsdauer am ïbrigen Dünndarm beträgt 2-3 Minuten, ausnahmsweise mehr, einmal 8 Minuten. Die Kalisalze haben genau dieselbe Wirkung wie am Duodenum. Die Natroncontraction ist immer gleichförmig circulär. In Bezug auf die Axe des Darmes ist sie bisweilen local, gewöhnlich erstreckt sich aber die Wirkung des Salzes mehr oder weniger deutlich etwa $1 / 2 \mathrm{~cm}$ nach oben. Dies zeigt sich in der Weise, dass ausser der localen Contraction nach oben einige rhythmische Contractionen auftreten, oder dass eine länger anhaltende zweite Contraction oberhalb entsteht, oder der Darm zusammenhängend sich aufwärts contrahirt. Es scheint immer das Streben nach einer aufsteigenden Contraction vorhanden zu sein, je nachdem aber der Darminhalt weniger oder mehr widersteht, kommt dieselbe wirklich zu Stande, oder sie wird nur durch die rhythmischen Contractionen angedeutet, oder es bleibt gar bei der localen Contraction.

Am Coecum und Dickdarm ist die Dauer der Contraction sehr unbestimmt, sie schwankt am Coecum zwischen 5 und 15 Minuten, am Dickdarm dauert sie bis 1 Stunde. 
Die Kaliumcontraction ist wie am ubrigen Darm eine starke Einschnürung genau der Reizstelle entsprechend, mehr oder weniger über den Umfang ausgedehnt. Am oberen Dickdarm, der mit consistentem breiigem Koth stark gefüllt ist, war die Contraction auffällig gleichmässig circulär, und der contrahirte Darm ging ziemlich allmählich in den normalen uiber (Fig. 3a). Reizt man das Coecum zwischen zwei Ligamenten, so erstreckt sich die Einschnïrung nie über dieselben hinaus, sondern die periphere Ausdehnung wird stets durch die beiden benachbarten Ligamente begrenzt.

Die Natriumsalze wirken stets, auch am Coecum, gleichmässig circulär ohne Rücksicht auf die Ligamente. Die Contraction erstreekt sich nach oben von der Reizstelle, oder tritt oberhalb derselben auf. Handelt es sich auch zuweilen nur um einige Millimeter, oder noch weniger, so ist die Erscheinung, wenn man sich nur die Reizstelle vorher genau notirt, z. B. mit einem Copirstift, der für sich nicht bemerkbar reizt, doch nicht zu iubersehen, und ganz constant zu beobachten.

Am Coecum liegt die grösste Tiefe der schmalen Einschnürung 3 bis 5 Millim. mehr nach oben, als der Reizstelle entsprechen wiirde. Das Coecum zeigt anscheinend wegen der Dünne der Wandungen und der Nachgiebigkeit des Inhaltes, alle Erscheinungen am schönsten und präcisesten vom ganzen Darm.

Im oberen Dickdarm gleicht der Contractionsring dem der Kaliumsalze, er liegt aber $1 / 2$ Millim. bis $1 / 2 \mathrm{~cm}$ höher, als der Reizstelle entspricht (Fig. 3 b).

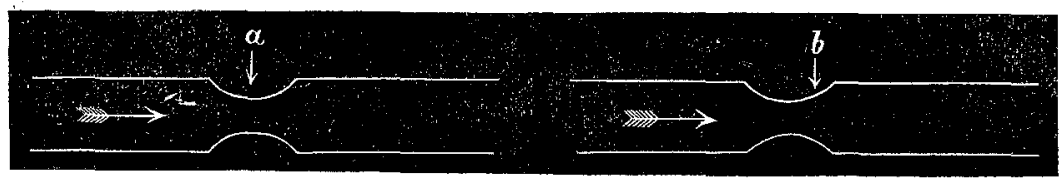

Fig 3.

Am unteren Dickdarm, wo die Kothballen ausgebildet sind, ist die Contraction in der Regel 1 bis $1 \frac{1}{2} \mathrm{~cm}$ breit, entsteht von der Reizstelle nach oben und persistirt an der Reizstelle am längsten. In der Gegend liegende Scybala werden gewöhnlich nach oben geschoben; widerstehen sie der Bewegung, so heben sie die Contraction mehr oder weniger auf. Durchschneidet man den Darm zwischen zwei Ballen, so wird der untere durch Reizung 
am unteren Ende nach oben entfernt, reizt man dagegen am oberen Ende des oberen, so tritt eine aufsteigende Contraction ein, ohne Einfluss auf den am Schnittende liegenden Kothballen ${ }^{1}$ ).

Die Harn- und Samenblase reagiren analog dem Magen. Die Contraction entspricht der Reizstelle bei beiderlei Salzen. Die $\mathrm{Na}$-Contraction ist ausgedehnter und weniger energiseh als die Ka-Contraction. Die Contractionsdauer ist fast constant 5 Minuten.

Bei einem Versuche, den ich an einer grossen weiblichen Ratte anstellte, erstickte das Thier wegen der nothwendigen festen Fixirung, als ich das Abdomen eben eröffnen wollte. Ich brachte die gewohnten $\mathrm{Ka}$ - und Na-Salze in üblicher Weise gleich nach dem Tode an den Darm, bemerkte aber keinerlei Wirkung. Ich halte es nicht für wahrscheinlich, dass die Salze gewirkt haben wiirden, wenn das Thier noch gelebt hätte, da ich mich öfter an anderen Thieren ïberzeugt habe, dass unmittelbar nach dem Tode die Empfindlichkeit gegen chemische Reize nicht herabgesetzt war. Die Eingeweide verhielten sich im Wasser wie ein mit Fett überstrichener Gegenstand, nahmen, so zu sagen, das Wasser schlecht an, und diese Eigenschaft derselben verhinderte es vielleicht, dass die sich bildende wässerige Salzlösung genügend einwirkte. Aehnliche Verhältnisse finden sich bei der Maus. Weitere Versuche konnten an Ratten leider nicht angestellt werden.

$\mathrm{Zu}$ den Experimenten am Mäusedarm verwandte ich weisse Mäuse, die gerade im Institut gehalten wurden. Die Reizung geschah mit Salzen in Substanz. Der Mäusedarm reagirte auf chemische Reize ziemlich träge.

Bei den Ka-Salzen beginnt die Contraction noch rasch, bei den Na-Salzen kann 1/2 Minute vergehen, oder die Reizung bleibt auch ganz erfolglos. Kommt einmal eine Wirkung zu Stande, so ist es ganz gleich, ob man längere oder kürzere Zeit gereizt hat. In der Regel gentigt eine Reizdauer von $1 / 4$ Minute, um eine Contraction hervorzurufen. Dieselbe nimmt dann langsam an Ausgiebigkeit zu, dann wieder ab und dauert einige Minuten. Die Ka-Contraction entspricht stets der Reizstelle und verbreitet sich nie gleichmässig circulär. Die Na-Contraction ist stets gleichmässig circulär.

1) Vgl. W. Preyer, Specielle Physiologie des Embryo, S. 320. 
Am Dünndarm entspricht der Na-Contractionsring fast immer genau der Reizstelle. Nur einmal wurde am Jejunum eine aufsteigende Contraction bemerkt, und einmal wurden am Duodenum schon vorher bestehende peristaltische Bewegungen oberhalb der Contraction stärker. Letzteres konnte ebensowohl eine Folge der Inhaltsstauung sein, als eine specifische Na-Wirkung. Am Coecum bewirkte das Na-Salz eine starke Contraction nach oben.

Am Dickdarm entsprechen die Erscheinungen denen beim Meerschweinchen, nur sind sie nicht ganz so ausgeprägt und constant. Am oberen Theile kommt eine schmale Einschnürung gewöhnlich bis $1 / 2 \mathrm{~cm}$ oberhalb der Reizstelle zu Stande, bisweilen entspricht die Einschnürung der Reizstelle. Am unteren Dickdarm pflegt der Contractionsring breiter zu sein, und erstreckt sich von der Reizstelle nach oben, oder doch weiter nach oben als nach unten.

Am Darmtractus des Hundes wirkten Ka- und Na-Salze in Substanz angewandt qualitativ sicher verschieden, aber nur am Coecum auffällig. Die Contraction war mehr oder weniger tief, nicht über $3 / 4 \mathrm{~cm}$ breit, war am Reizpunkte am stärksten und erstreckte sich über einen grösseren oder kleineren Theil der Peripherie. Sie fiel bei den Ka-Salzen ziemlich energisch aus und kam bei einer Berührung von $1 / 8$ Minute stets zu Stande, bei den Na-Salzen etwas schwächer; es war öfter eine Berührung von $1 / 4$ Minute und mehr nöthig, um überhaupt eine Wirkung hervorzubringen. Der Hundedarm gehört zu den auf chemische Reize weniger empfindlichen. Die Contraction beginnt öfters erst $1 / 2$ Minute nach der Reizung, nimmt dann $1 / 4$ bis $3 / 4$ Minuten zu und ist nach 1 bis 2 Minuten allmählich wieder verschwunden. Während man bei anderen Thieren, z. B. beim Meerschweinchen, schon am Bilde der Zusammenziehung mit Leichtigkeit sehen kann, ob es sich um eine $\mathrm{Ka}$ - oder $\mathrm{Na}$-Contraction handelt, ist dies beim Hundedarm nicht wohl möglich. Die versehiedene Wirkung! äussert sich nur an dem Orte der Zusammenziehung. Die Ka-Contraction entspricht stets dem Orte der Reizung, die Na-Contraction erstreckt sich am Coecum rom Reizpunkte $3 / 4 \mathrm{~cm}$ nach oben, das heisst nach dem blinden Ende zu (Fig. 4), am übrigen Darme kommt sie 1-2 $\mathrm{mm}$ höher oben zu Stande, als dem Reizpunkte entspricht (Fig. 5). Nach dem Tode treten erst schwächere, dann stärkere Darmbewegungen ein, Reizversuche während der Dauer derselben, hatten den gleichen 
Erfolg wie vorher. Nach etwa $3 / 4$ Stunden hörten die Darmbewegungen auf, genau mit ihnen die Na-Wirkung. Die Ka-Wirkang war noch unverändert.

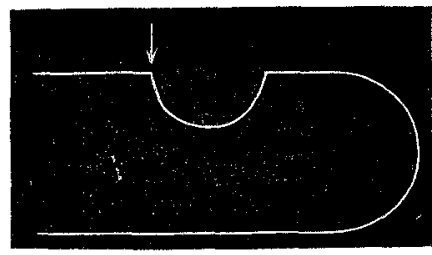

Fig. 4.

Coecum nach Reizung mit Natriumnitrat.

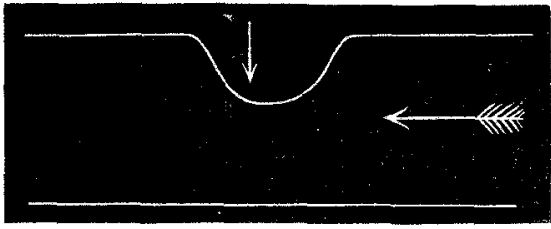

Fig. 5.

Diekdarm des Hundes mit Natriumnitrat gereizt.

Die Experimente am Darme des lebenden Huhnes wurden dadurch ermöglicht, dass der Vogel nach der Tracheotomie durch künstliche Respiration am Leben erhalten wurde.

Die Salze wurden in Substanz angewandt. Die Ka-Salze bewirkten eine axial und peripher locale Contraction von etwa 6 Minuten Dauer und $1 / 2$ bis $1 \mathrm{~cm}$ Breite. (Fig. 6).

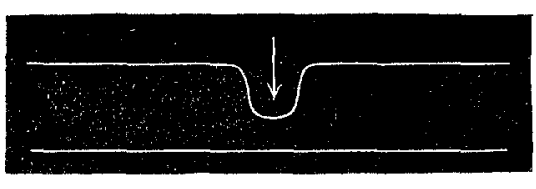

Fig. 6.

Hühnerdarm in der Nähe des

Magens nach Reizung mit Kaliumnitrat.

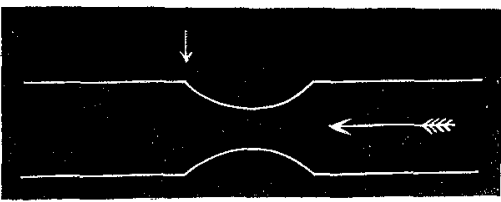

Fig. 7.

Hühnerdarm in der Nähe des Magens nach Reizung mit Natriumnitrat.

Die Na-Salze verursachen eine circuläre von der Reizstelle nach oben sich erstreckende Contraction. Dieselbe ist etwa $1 / 2 \mathrm{~cm}$ breit und hat eine Dauer von ungefähr 2 Minuten. Die Contraction verschwindet allmählich, zuletzt an der Reizstelle. (Fig. 7).

Die postmortalen Darmbewegungen danern etwa 10 Minuten, ebensolang reagirt der Darm charakteristisch auf Natriumverbindungen. Die Ka-Salze wirken noch 10 Minuten nach Aufhören der Darmbewegungen wie vorher.

Bei den Versuchen an Fröschen hatte das Wasser die Temperatur des Zimmers. Die Salze wurden in Substanz applicirt, in der Regel 1/4 Minute lang. Bei dem ausserordentlich langsamen Verlauf der Erscheinungen war es bisweilen schwer, zu entscheiden, 
ob dieselben spontan auftraten, oder durch die Salze verursacht wurden. Ich erwähne hier nur, was für eine wirkliche Folge der Salzeinwirkung mit Wahrscheinlichkeit zu halten ist. Die Reizung bleibt bisweilen, besonders bei den Na-Salzen, ohne Wirkung, oder die gereizte Stelle wird anämisch. In der Regel erfolgt eine Contraction. Diese zeigt sich schon während der Reizung, oder es vergeht eine kürzere oder längere Zeit, bei den Ka-Salzen bis 1 Minute, bei den Na-Salzen bis 5 Minuten. Die Contraction ist in der Regel nach einer Daner von etwa 5 Minuten verschwunden, doch kann die Wirkung der Reizung auch bis über 1 Stunde bestehen. Statt einer gleichmässigen Contraction erhielt ich einmal nach Reizung des Magens mit Natriumnitrat und des Duodenums mit Kaliumnitrat eine Contraction von 15 Minuten in der Weise, dass dieselbe am Magen $12 \mathrm{mal}$, am Duodenum 15 mal deutlich stärker und schwächer wurde, beziehungsweise verschwand.

In Bezug auf die axiale Localisation wurde nach Reizung des unteren Darmes mit Natriumnitrat zweimal Contraction $1 / 4 \mathrm{~cm}$ oberhalb der Reizstelle deutlich bemerkt (Fig. 8).

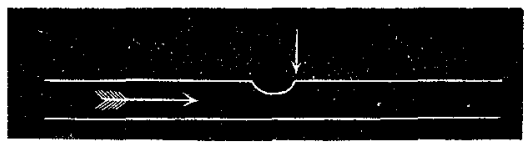

Fig. 8.

Auch die in Fig. 9 dargestellten Erscheinungen am Magen nach Reizung mit demselben Salze würden ähnlich aufzufassen sein.

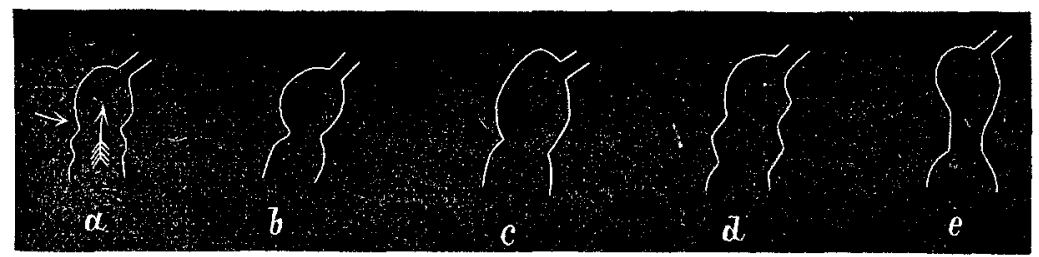

Fig. 9.

a stellt den unteren Magen 2 Minuten nach Reizung mit Natriumnitrat dar, b 4, c 10, d 20, e 25 Minuten nach der Reizung. 30 Minuten nach der Reizung war das Bild wieder wie in a und nach 40 Minuten glaubte ich immer noch eine schwache Contraction zu bemerken.

Bei den übrigen zahlreichen Reizversuchen mit Natriumsalzen zeigte sich wie bei den Kaliumsalzen, dass die Contraction axial genau der Reizstelle entspricht und bis $1 / 2 \mathrm{~cm}$ Breite erreicht. 
In Bezug anf die Cireumferenz des Darmes bleibt die Contraction bei beiderlei Salzen ganz local, oder ist gleichmässig circulär, oder die Wirkung liegt zwischen diesen beiden Extremen.

Halten wir die Erscheinungen nach Anwendung von Ka- und Na-Salzen am Froschdarme zusammen, so zeigt sich, dass die ersteren sicherer und schneller wirken als die letzteren. Ein nur irgendwie constanter qualitativer Unterschied des Erfolges nach Reizung mit den einen oder den anderen Salzen ist nicht zu ersehen.

Einige Versuche, die ich mit Salzen in Substanz an Embryonen ${ }^{1}$ ) und jüngeren Thieren anstellte, ergaben, dass die KaContraction im Ganzen der Wirkung am erwachsenen Thiere gleicht. Die Na-Salze wirken gar nicht, oder wie die Ka-Salze, nur sehwächer, oder es zeigt sich mehr oder weniger deutlich die NaReaction der erwachsenen Thiere. In der Regel zeigt erst im postembryonalen Leben der Darm nach und nach seine eigenthümliche Reaction auf Reizung mit Na-Salzen wie beim erwachsenen Thiere.

Die speciellen Versuchsergebnisse sind folgende:

Bei 11 nahezu reifen Meerschweinchenembryonen reagirte der Darm von 9 Thieren auf $\mathrm{Na}$-Salze entweder wie auf Ka-Salze oder es zeigten sich verwischt und undeutlich die Erscheinungen, die wir beim erwachsenen Meerschweinchen gefunden haben. Bei zwei fast reifen Embryonen reagirte der Darm in ganz präciser Weise analog dem erwachsenen Thiere. Beide Embryonen stammten von demselben Mutterthier; an dem einen wurden Reizversuche angestellt, bevor es geathmet hatte. Die Resultate waren an beiden Embryonen ganz gleich.

Von zwei neugeborenen Kaninchen reagirte der Darm des einen anf Na-Salze nicht. Bei dem anderen zeigte sich am Duodenum in mehr, am übrigen Dünndarm in minder ausgesprochener Weise, die dem erwachsenen Kaninchen eigene Na-Contraction. Am Dickdarm wirkten die Na-Salze wie Ka-Salze.

Bei einem 14 Tage alten Hunde wirkten die Na-Salze qualitativ ganz wie Ka-Salze. Die Contraction, etwa 5 bis $7 \mathrm{~mm}$ lang, entspricht genau der Reizstelle und erstreckt sich nicht uber den

1) Dass nach mechanischer, electrischer, chemischer Reizung des fötalen Dünn- und Dickdarmes vom Meerschweinchen locale Constrictionen eintreten, zeigte Prof. Preyer in seiner "Speciellen Physiologie des Embryo" p. 319. 
ganzen Umfang des Darmes. Bei den Ka-Salzen wirkt eine momentane Berührung, bei den Na-Salzen muss die Berührung bis 15 Sekunden anhalten. Die Dauer der Berïhrung hat auf die Ausgiebigkeit der Contraction keinen Einfluss. Nach etwa 2 bis 3 Minuten sind die Contractionen wieder verschwunden.

In einem eigenthümlichen Zusammenhange mit der Entwickung der Na-Reaction scheinen die Darmbewegungen zu stehen, die unmittelbar vor oder gleich nach dem Tode der Thiere eintreten und längere Zeit, beim Meerschweinchen bis über 2 Stunden, bestehen. Die Fähigkeit zu postmortalen Darmbewegungen entwickelt sich neben der Reaction anf Na-Salze so, dass die Entwicklung der specifischen Na-Reaction etwas fribher beginnt.

$\mathrm{Zu}$ der angefuihrten Annahme führte folgendes:

Bei allen Embryonen und jüngeren Thieren, die zu den eben beschriebenen Versuchen dienten, fehlten die postmortalen Darmbewegungen noch vollständig, während die Na-Reaction bisweilen schon angedeutet war. Nur bei zwei Meerschweinchenembryonen zeigten sich $1 / 4$ Stunde lang deutliche postmortale Bewegungen, und das waren gerade jene zwei einzigen Embryonen, bei denen die Na-Reaction praecis wie am erwachsenen Thiere sich zeigte. Zwei noch nicht ausgewachsene Katzen (320 grm schwer), welche die oben erwähnte Natrium-Contraction nur unvollständig zeigten, liessen auch nur am Dünndarm und nur ganz schwache postmortale Darmbewegungen beobachten im Gegensatze zu der erwachsenen Katze.

Nach dem Tode des erwachsenen Thieres zeigt sich die Natriumreaction genau so lange wie die Darmbewegungen, während die Kaliumreaction noch längere Zeit unverändert besteht.

Das Gesammtergebniss meiner Versuche ist sehr einheitlich und lässt sich kurz zusammenfassen:

Bei allen zur Untersuchung gekommenen erwachsenen Warmblittern ist die Wirkang der Natriumsalze auf den Darmtractus qualitativverschieden von derder Kaliumsalze. Der Versuch mit der Ratte kommt als resultatlos nicht in Betracht. Der genannte qualitative Unterschied ist aber sehr verschieden ausgeprägt bei den verschiedenen Thierarten. Die wechselnden 
Erscheinungen bei den einzelnen Thierarten beruhen fast nur auf der verschiedenartigen Wirkung der Natriumsalze. Die Reizung mit Kaliumsalzen hat überall ziemlich das gleiche Resultat: Contraction entsprechend der Reizstelle, mehr oder weniger weit und gleichmässig über den Umfang des Darmes ausgedehnt.

Die specifische Natriumreaction entwickelt sich im spätembryonalen oder auch erst im postembryonalen Leben.

Die Natriumsalze wirken nicht genau der Reizstelle entsprechend, die Localisation der Contraction wechselt bei den einzelnen Thierarten.

Bei dem Kaninchen, an dem die Natriumreaction entdeckt und zuerst beschrieben wurde, ist dieselbe auch am auffälligsten, weniger schon bei der Katze.

Auch beim Meerschweinchen sieht man sofort an der Art der Zusammenziehung, ob mit einem Kalium- oder Natriumsalz gereizt worden ist. Die Kaliumcontraction ist fast nie, die Natriumcontraction stets gleichmässig über den Umfang des Darmes ausgedehnt. Ausserdem zeigt sich am Dünndarm mehr oder weniger deutlich eine Wirkung der Natriumsalze nach oben in Form einer aufsteigenden Contraction, oder einer zweiten Contraction oberhalb, die rhythmisch verschwinden und wiederkehren kann. Am Coecum und oberen Dickdarm liegt die Contraction stets oberhalb der Reizstelle, am unteren Dickdarm erstreckt sie sich von der Reizstelle nach oben.

Bei der Maus verhält sich der Dickdarm ganz ähnlich, wie beim Meerschweinchen. Am Coecum zeigt sich eine aufsteigende Contraction, Am Dünndarm ist die Natriumreaction scharf gekennzeichnet dureh eine gleichmässig circuläre Ausdehnung der Contraction, die das Kaliumsalz hier nie hervorruft.

Am Hundedarm war die verschiedene Wirkung der Kaliumund Natriumsalze am schwersten zu sehen. Die letzteren wirken hier ebensowenig gleichmässig circulär wie die Kaliumsalze. Die Contraction befindet sich jedoch nach Reizung mit Natriumsalzen 1 bis $2 \mathrm{~mm}$ mehr nach oben, als der Reizstelle entsprechen würde, am Coecum geht die Contraction von der Reizstelle $3 / 4 \mathrm{~cm}$ nach oben.

Das Huhn gewährt wieder klarere Erscheinungen: $1 / 2$ bis $1 \mathrm{~cm}$ breite, nicht circuläre, der Reizstelle entsprechende Kaliumcon- 
traction, $2 \mathrm{~cm}$ breite, gleichmässig circuläre, von der Reizstelle nach oben sich erstreckende Natriumcontraction.

Die Reizung von Magen, Harn- und Samenblase ergab bei keinem Thiere einen qualitativen Unterschied der beiden Salze. Die Natriumsalze wirken etwas schwächer und vielleicht diffuser als die Kaliumsalze.

Die Ergebnisse der Versuche am Froseh sind nicht einheitlich zusammenzufassen.

In Rücksicht auf das Bild der Contraction, wie es beim Kaninchen und ähnlich bei der Katze sich darbietet, hat sich ergeben, dass dasselbe bis jetzt auf kein anderes Thier zu übertragen ist. Die Annahme, dass man etwa durchweg ein ganz analoges Bild erwarten dürfe, hat sich als unhaltbar herausgestellt. Warum insbesondere die Erscheinungen am Kaninchen auf den Menschen durchaus nicht übertragbar sind, werde ich noch erwähnen. Nur soweit lässt sich die Natriumreaction des Kaninchendarmes verallgemeinern, dass bei allen Warmblütern, die zu meinen Experimenten benutzt wurden, ebenfalls eine specifische Natriumreaction besteht. Dieselbe kann allerdings mehr oder weniger an die beim Kaninchen erinnern.

Meine allgemeine Erklärung für die Eigenthümlichkeit der Kali- und Natriumreaction ist jedoch ganz ähnlich der, wie sie Nothnagel für das Kaninchen und die Katze angab. Meine Versuche sind geeignet, die Vermuthung des genannten Forschers wahrscheinlicher zu machen.

Nothnagel nimmt an, dass durch die Kaliumsalze direct die Muskeln gereizt werden, dass bei den Natriumsalzen ausser einer directen Muskelreizung eine Reizung nervöser Apparate stattfinde, und dass auf letzterer die specifische Natriumcontraction beruhe.

Die Angabe für die Kaliumsalze kann ich für meine sämmtlichen Versuche gelten lassen; um aber den Umstand zu erklären, dass die Kaliumsalze bisweilen eine ganz gleichmässig circulär ausgedehnte Contraction verursachen, wie am oberen Dickdarm des Meerschweinchens, glaube ich auch den Kaliumsalzen die Fähigkeit einer nervösen Erregung zusprechen zu miissen.

Als Beweis für die Annahme, dass die specifische Natriumerscheinung auf einer nervösen Erregung beruhe, nimmt Noth- 
nagel in erster Linie an, dass die Contraction nicht der Berïhrungsstelle entspricht, und dass der Kaninchendarm nach Aufhören der postmortalen Bewegungen die Natriumreaction verloren hat. Beide Gründe lassen sich für meine sämmtlichen Warmblüter' anführen, der letztere in noch bestimmterer Weise. Die specifische Natriumreaction tritt, wie ich gefunden habe, im embryonalen oder postembryonalen Leben ganz gleichzeitig auf mit der Fähigkeit des Darmes zu postmortalen Bewegungen. Im früheren Leben unterscheidet sich die Natriumcontraction qualitativ nicht von der Kaliumcontraction. In derselben Minute in der die postmortalen Darmbewegungen aufhören, ist es anch mit der specifischen Natriumreaction aus, während die Kaliumsalze noch ganz präcis wirken.

Die Ursache für das wechselvolle Bild der Natriumcontraction wäre wohl in einer verschiedenen Verlaufsrichtung der betreffenden Nervenelemente in den verschiedenen Darmabschnitten eines Thieres einerseits und dem Darme der verschiedenen Thiere anderseits zu suchen. Wie man sich die Sache des Näheren zu denken hat, dariiber wage ich kein Urtheil abzugeben.

Dass den Natriumsalzen auch eine directe Muskelreizung zuzuschreiben ist, geht schon daraus hervor, dass der Darm, der noch nicht die Fähigkeit zu postmortalen Darmbewegungen, und somit, wie ich annehme, noch nicht die durch die Natriumverbindungen erregbaren Nervenelemente besitzt, durch Natriumsalze in gleicher Weise verändert wird, wie durch Kaliumsalze. Beim erwachsenen Thiere kann man bisweilen deutlich eine locale, wahrscheinlich muskuläre, und eine oberhalb befindliche, wahrscheinlich nervöse Reaction unterscheiden (Fig. 10). Auch der Umstand

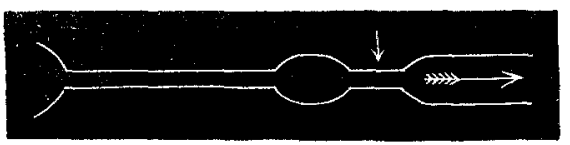

Fig. 10.

Meerschweinchen-Duodenum nach Reizung mit Natriumnitrat.

dass nach Eintreten einer aufsteigenden Natriumcontraction, die der Reizung entsprechende Stelle öfter länger contrahirt bleibt, als das iubrige Darmstïck, dürfte hier anzuführen sein.

Eigenthümlich ist es, dass der Menschendarm allein auf Natriumsalze anders reagirt als der Darm sämmtlicher angeführter 
Warmblïter zusammengenommen. Hier fast immer Wirkung nach oben, nie nach abwärts, beim Menschen locale Contraction mit Constrictionen oberhalb und unterhalb.

Ich muss entschieden annehmen, dass die gleich nach dem Tode angestellten Versuche Bardelebens dieselbe Gültigkeit haben, als wären sie am lebenden Menschen vorgenommen. Es wird in dem einen Falle besonders hervorgehoben, dass während der Versuche Darmbewegungen bestanden, und die Erfahrung lehrt, dass die Natriumreaction unverändert bleibt, bis die Darmbewegungen aufhören. In dem anderen Falle schlug sogar das Herz noch, und die Körpertemperatur war noch normal. Dass etwa eine spontane Zusammenziehung des Darmes mit einer durch das Salz veranlassten verwechselt worden wäre, oder dass weniger auffällige specifische Eigenschaften der Natriumreaction ubersehen worden wären, wird man nicht glauben. Man muss also wohl dabei stehen bleiben, dass sich der Verlauf oder die Erregbarkeit der Nervenelemente, die bei der Reizung des Darmes mit Natriumsalzen in Betracht kommen, beim Menschen anders verhalten, als bei den von mir beobachteten Thieren.

Herr Professor Pr e yer machte mich darauf aufmerksam, dass der Grund dieses abweichenden Verhaltens vielleicht in dem Umstande liege, dass der Mensch von Kindheit an durch den reichlicheren Genuss von Kochsalz den Darm mehr mit Natriumverbindungen in Berührung bringe, als dies bei anderen Thieren der Fall ist. Die Vermuthung hat viel Wahrscheinliches, und eine Entscheidung liesse sich vielleicht mit Hülfe. von Fütterungsversuchen durch Thierexperimente ermöglichen. 